\title{
Editorial for Advances in Intelligent Mobile Applications Special Issue
}

\author{
Jordán Pascual Espada ${ }^{1} \cdot$ Ronald R. Yager $^{2} \cdot$ Antonio J. Jara $^{3}$
}

Published online: 3 March 2016

(C) Springer Science+Business Media New York 2016

\section{Editorial}

This special issue features seven selected papers with high quality related to intelligent mobile applications. The papers included in this special issue have been selected among 30 papers.

The first paper, "A POI-aware Power Saving Scheme for Ubiquitous Touring Service using Mobile Devices over the Cellular and Wi-Fi Hybrid Network", authored by ChaoHsien Lee, Chung-Ming Huang and Wei-Shuang Chen presents PETS "Power Efficient Touring Scheme". This research work considers the peculiarities of a pedestrian touring service and concentrates on how to save battery power of mobile devices smartly. The system is able to dynamically adjust the GPS sampling rate with the help of embedded sensors based on the relationship among the current geo-location, motion behavior and physical environment, this scheme downloads neighboring POIs using cellular network. PEST considers communication restrictions of heterogeneous wireless technologies, including cellular network and Wi-Fi.

Jordán Pascual Espada

pascualjordan@uniovi.es

Ronald R. Yager

yager@panix.com

Antonio J. Jara

antonio.jara@hevs.ch

1 Computer Science Department, University of Oviedo, Oviedo, Spain

2 Machine Intelligence Institute, Iona College, New Rochelle, NY, USA

3 University of Applied Sciences Western Switzerland, Delémont, Switzerland
The second paper, "Intelligent mobile system for improving spatial design support and security inside buildings", authored by Janusz Bedkowski, Karol Majek, Piotr Majek, Pawel Musialik, Michal Pelka and Andreas Nuechter presents an Intelligent mobile application for spatial design support and security domain, specially designed for protection of urban environments including critical infrastructures. The system defines a Complex Shape Histogram and an artificial intelligence engine, used for classifying 3D point clouds with Support Vector Machine. The Complex Shape Histograms is used for loop closing detection in the simultaneous localization and mapping algorithm. The proposed system was tested in the Museum of the History of Polish Jews (Warsaw, Poland), and Airport in Łódź, Poland.

The third paper "NFC and Cloud-based Lightweight Anonymous Assessment Mobile Intelligent Information System for Higher Education and Recruitment Competitions" authored by Sergio Rios-Aguilar, Jordán Pascual Espada and Ruben Gonzalez Crespo. This research work proposes a solution to the Anonymous Assessment problem. NFC Secured Exams, is a low cost and secure anonymous assessment solution in which the data is digitally encrypted and inserted in NFC tags so that only the system can relate that digital information with the corresponding student. The system is useful to guarantee the students anonymity, reduce the time spent handing in the exams and increasing the overall efficiency of requesting exam reviews. The solution can combines the NFC technology with the S-Pen technology or a capacitive stylus in a double validation process.

The forth paper "Framework for Enhancing Mobile Availability of RESTful Services: A Connectivity-Aware and Risk-Driven Approach" authored by Shang-Pin Ma, Wen-Tin Lee, Ping-Chang Chen and Chi-Chia Li. Authors propose a connectivity-aware, risk-driven (CARD) approach to the delivery of RESTful services. The CARD framework is proposed as a means of enhancing the mobile availability of RESTful services for smartphones under a variety of wireless 
network conditions. CARD responds to current network conditions by performing prefetch service responses and using cached services when connected to a high speed wireless network, using cached services when the network is unstable, and allowing the user to request cached services from other users when no wireless network is available.

The fifth paper "GreenBikeNet: An Intelligent Mobile Application with Green Wireless Networking for Cycling in Smart Cities" authored by Osama M.F. Abu-Sharkh1 and Zaid Dabain. The paper presents a mobile application with intelligent systems to provide services for cyclists and enhance their cycling experiences in Smart cities. The application uses smartphones and low-power ZigBee technology for ad-hoc networking. Cyclists can join either public networks or create their own private networks to exchange information and utilize several developed services, like voice communication and intelligent geo-localization systems which uses smartphone's GPS receiver to provide real-time geo-locations and speeds of all cyclists connected to the same network on a visual interactive map.

The sixth "Multi-hop Mobility Prediction" authored by Zhiyong Yu, Zhiwen Yu and Yuzhong Chen. This paper propose a novel algorithm to expand the application value of mobility prediction and resolve the limitation of one-hop mobility prediction. The authors define a multi-hop mobility prediction system and propose a firstorder Markov-based MHMP algorithm (Markov-MHMP). In this algorithm multiple feature sets are considered to construct mobility transition matrices, along with user's current location and other context, so that we can figure out the user's location probability distribution for a long time thereafter. This system can be useful to predicting user encounters for advanced phone applications in mobile crowd sensing and opportunistic networks.

The last paper "Intelligent Sensing for Citizen ScienceChallenges and Future Directions" co-authored by Michael J. O'Grady, Conor Muldoon, Dominic Carr, Jie Wan, Barnard Kroon and Gregory M. P. O'Hare. This paper outlines how the disparate technologies of sensors and mobile Apps may be integrated. Specifically, services provided by an arbitrary sensor may be accessed via a Cordova plug-in. Such services invariably include discovery, connection establishment and data upload amongst others. In abstracting and encapsulating such functionality as a plug-in, sensor services can be seamlessly integrated into a variety of third-party apps. Such an approach protects the software developer from the intricacies of sensor platforms, whilst broadening the availability of sensor services to the software developer community.

The guest editors are thankful to our reviewers for their effort in reviewing the manuscripts. We also thank the Editin-Chief, Dr. Imrich Chlamtac for his supportive guidance during the entire process.

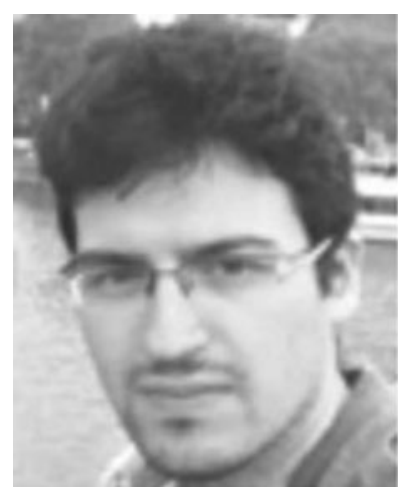

Dr. Jordán Pascual Espada Associate Professor at Computer Science Department of the University of Oviedo. Ph.D. ( Cum Laude) from the University of Oviedo in Computer Engineering B.Sc. in Computer Science Engineering and a M.Sc. in Web Engineering. $\mathrm{He}$ has published more than 30 articles in international journals and conferences, he has worked in several relevant research projects related to RFID, Internet of Things and Cloud computing. He participates as editorial board member in few international journals like International Journal of Interactive Multimedia and Artificial Intelligence, Communications and Network and International Journal of Handheld Computing Research, each others. His research interests include the Internet of Things, Smart Cities, exploration of new applications and associated human computer interaction issues in ubiquitous computing and emerging technologies, particularly mobile and Web applications.

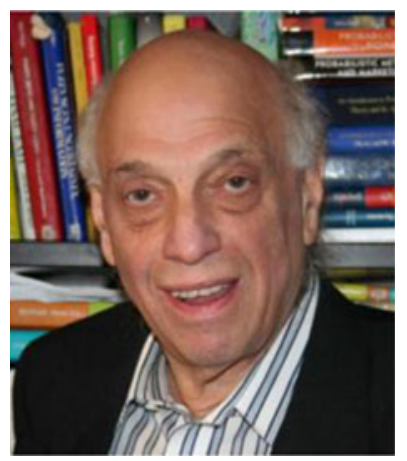

Dr. Ronald R. Yager is Professor of Information Systems and Director of the Machine Intelligence Institute at Iona College. He is among the world's most highly cited researchers with over 47,000 citations to his work in Google Scholar. He is editor and chief of the International Journal of Intelligent Systems and serves on the editorial board of numerous journals. He has published over 500 papers and edited over 30 books in areas related to fuzzy sets, computational intelligence, human behavioral modeling, decision-making under uncertainty and the fusion of information. He is the 2016 recipient of highly prestigious IEEE Frank Rosenblatt award. He was the recipient of the IEEE Computational Intelligence Society Pioneer award in Fuzzy Systems. He received the special honorary medal of the 50th Anniversary of the Polish Academy of Sciences. He received the Lifetime Outstanding Achievement Award from International the Fuzzy Systems Association. He received honorary doctorate degrees, honoris causa, from the Azerbaijan Technical University and the State University of Information Technologies, Sofia Bulgaria. Dr. Yager is a fellow of the IEEE, the New York Academy of Sciences and the Fuzzy Systems Association. He has served at the National Science Foundation as program director in the Information Sciences program. He was a NASA/ Stanford visiting fellow and a research associate at the University of California, Berkeley. He has been a lecturer at NATO Advanced Study Institutes. He is a visiting distinguished scientist at King Saud University, Riyadh Saudi Arabia. He is an adjunct professor at Aalborg University in Denmark. He received his undergraduate degree from the City College of New York and his Ph. D. from the Polytechnic Institute New York University. 


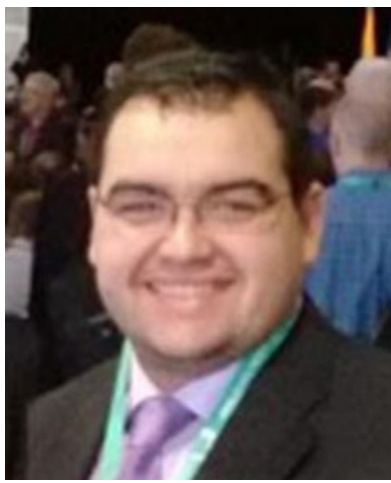

Dr. Antonio J. Jara Assistant Prof. PostDoc at University of Applied Sciences Western Switzerland (HES-SO) from Switzerland, vice-chair of the IEEE Communications Society Internet of Things Technical Committee, and founder of the Wearable Computing and Personal Area Networks company HOP Ubiquitous S.L., He did his $\mathrm{PhD}$ (Cum Laude) at the Intelligent Systems and Telematics Research Group of the University of Murcia (UMU)

from Spain. He received two M.S. (Hons. - valedictorian) degrees. Since 2007, he has been working on several projects related to IPv6, WSNs. and RFID applications in building automation and healthcare. He is especially focused on the design and development of new protocols for security and mobility for Future Internet of things, which was the topic of his Ph.D. Nowadays, he continues working on IPv6 technologies for the Internet of Things in projects such as IoT6, and also Big Data and Knowledge Engineering for Smart Cities and eHealth. He has also carried out a Master in Business Administration (MBA). He has published over 100 international papers, As well, he holds one patent. Finally, he participates in several Projects about the IPv6, Internet of Things, Smart Cities, and mobile healthcare. 